\title{
A New Angle-Based Location Method Using a Forward-Link Signal
}

\author{
Seung-Hun Song, ${ }^{1}$ Hyun-Ja Im, ${ }^{1}$ Ji-Won Park, ${ }^{1}$ and Tae-Kyung Sung ${ }^{2}$ \\ ${ }^{1}$ Department of Information and Communication Engineering, Chungnam National University, Daejeon 305-764, South Korea \\ ${ }^{2}$ Division of Electrical and Computer Engineering, Chungnam National University, Daejeon 305-764, South Korea
}

Correspondence should be addressed to Tae-Kyung Sung, tksaint@cnu.ac.kr

Received 14 April 2009; Accepted 23 June 2009

Recommended by Magnus Jansson

With conventional AOA positioning using a reverse-link signal, each sensor is equipped with an array antenna to measure the incident angle of the signal emitting by a mobile source. In order to perform the complicated array processing for angle measurements, both the sensor size and the power consumed by the sensor can increase in RLAOA (reverse-link AOA) positioning. In some applications such as mobile vehicle localization, the vehicle has fewer limitations in terms of size or power consumption. Rather, it is desirable to make the sensor as light as possible. This paper presents a new angle-based positioning scheme using a forward-link signal. Under the assumption that a ground vehicle moves on a horizontal surface, a measurement equation for the FLAOA (forward-link AOA) is initially derived. Using the measurement equation, a closed-form solution for FLAOA positioning is proposed. With the proposed method, it is also possible to estimate the azimuth of the vehicle as well as its position. The performance of the proposed method is compared to that of RLAOA positioning in a computer simulation. The simulation results show that the proposed method is potentially suitable for applications involving the localization and guidance of mobile vehicles.

Copyright (C) 2009 Seung-Hun Song et al. This is an open access article distributed under the Creative Commons Attribution License, which permits unrestricted use, distribution, and reproduction in any medium, provided the original work is properly cited.

\section{Introduction}

Over the past few decades, many types of radio-navigation systems have been developed. Examples include GNSS (global navigational satellite system), AGNSS (assisted GNSS), and geolocation systems using cellular networks. These radio-navigation systems are widely used in various LBS (location-based service) applications. To improve or complement the existing systems, upgraded or new location systems are currently under development. Examples of this include GNSS modernization and IR-UWB (impulse radio ultra wide band) WPAN (wireless personal area network) location systems [1-4].

Depending on the measurements used, radio-navigation systems can be classified as either time based or angle based. An OWR (one-way ranging) TOA (time-of-arrival) system measures the signal propagation time between the sensor and a user, whereas a TDOA (time-difference-of-arrival) system uses the time difference between different sensors. As sensors are installed at known positions, the three-dimensional user position can be estimated from at least four TOA measurements, or equivalently, TDOA measurements from at least three. In time-based OWR, either a forward-link or a reverse-link signal can be used. Using a forward-link signal from the sensor to the user, a user estimates its position on a self-positioning basis. Using a reverse-link signal, remote positioning or indirect positioning is possible. Although time-based OWR systems require the timing synchronization of sensors with a very high precision, they are able to offer a high degree of positioning accuracy [3-7].

AOA (angle-of-arrival) systems measure the incident angle of a signal from a source at an array antenna [5-7]. They can achieve high positioning accuracy without timing synchronization. In conventional AOA systems, sensors installed at known positions are equipped with array antennas to measure the incident angle of the reverse-link signal from the user. In this RLAOA (reverse-link AOA) positioning system, angle measurement is a function of the unknown user position. From at least three RLAOA measurements, three-dimensional user position can be estimated. In RLAOA 


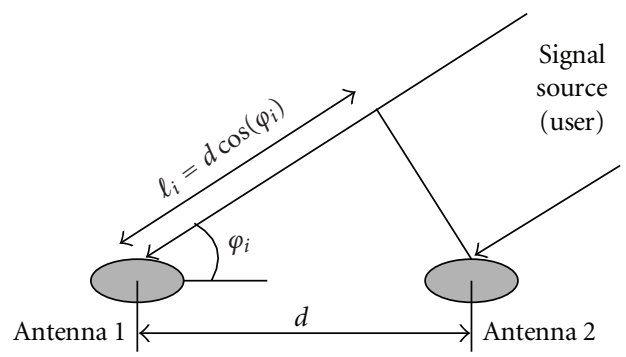

Figure 1: AOA measurement at an array antenna.

positioning, both the size and power consumption of the sensors can increase owing to the size of the array antenna and due to the complicated array signal processing for the angle measurement.

In some applications, such as mobile vehicle localization, it is often preferred to have sensors run on batteries. On the other hand, the vehicle has fewer limitations in terms of size or power consumption. To meet these requirements, it is desirable that the array antenna is installed on the vehicle to measure the incident angle of the forward-link signal emitted by the sensors. In this FLAOA (forward-link AOA) approach, however, angle measurement is influenced by unknown user attitude and position. Consequently, more than six angle measurements are necessary to estimate threedimensional user position and attitude. Moreover, FLAOA measurements are highly nonlinear with respect to the user position and attitude. As FLAOA measurement equations are highly nonlinear and very complicated, FLAOA positioning has been rarely studied.

As RLAOA measurements are nonlinear with respect to the user position, NLS (nonlinear least square) approaches have been proposed to get the position estimates [8]. Examples include the GN (Gauss-Newton) method [3, 5, 6] and closed-form solutions $[7,9,10]$. The GN method can achieve high accuracy, but the convergence of the iterative process is not always ensured if the initial guess is not sufficiently accurate. The closed-form solution provides a noniterative solution that avoids the convergence problem; moreover, its computational load is light. However, its estimation error is somewhat larger compared to that of the GN method where GN converges.

This paper presents a new AOA location methodology using FLAOA measurements. Under the assumption that a ground vehicle moves on a horizontal surface, FLAOA measurement is initially modeled. Using an FLAOA measurement equation, a closed-form solution for FLAOA positioning is newly proposed. Given that the proposed method estimates the azimuth of the vehicle as well as its position, additional information pertaining to the guidance and control can be provided. In the guidance of ground vehicles, the azimuth should be provided to control the moving direction and consequently to make the vehicle arrive at a desired destination. Through a computer simulation, the performance of the GN method and the closed-form solution for FLAOA measurements is analyzed. The performance of FLAOA positioning is then compared to that of RLAOA positioning. Finally, an efficient location estimation method for a mobile vehicle is suggested.

\section{FLAOA Positioning for Mobile Vehicles}

2.1. FLAOA Measurement Model. AOA location systems measure the incident angle at an array antenna. Assuming that two antennas are horizontally separated by a distance $d$ to form an array antenna, as shown in Figure 1, through the cross-correlating signals received at Antenna 1 and 2, the path difference $l_{i}$ can be obtained [11]. From the path difference, the incident angle $\varphi_{i}$ is given by

$$
d \cos \left(\varphi_{i}\right)=l_{i}, \quad 0<\varphi_{i}<\pi .
$$

In conventional RLAOA location systems, sensors are equipped with array antennas to obtain angle measurements using the reverse-link signal from a user. Figure 2(a) shows the basic principles of RLAOA for a two-dimensional case. In the figure, $\left\{x_{R}, y_{R}\right\}$ denotes a fixed reference frame. Assuming that orientations of array antennas in all sensors are set to 0 with respect to the reference frame, the RLAOA measurement is represented by

$$
\tan \left(\varphi_{i}\right)=\frac{y-y_{i}}{x-x_{i}}
$$

where $(x, y)$ and $\left(x_{i}, y_{i}\right)$ denote the user and the $i$ th sensor position with respect to the reference frame, respectively. As an RLAOA measurement is a function of the user position, a two-dimensional user position can be estimated from at least two RLAOA measurements.

In FLAOA positioning, an array antenna is installed on the vehicle (user), as shown in Figure 2(b). In the figure, $\left\{x_{R}, y_{R}\right\}$ and $\left\{x_{U}, y_{U}\right\}$ denote the reference frame and the user frame, respectively. The user frame is rigidly attached to the vehicle, and the $\mathrm{x}$-axis is parallel to the baseline vector between two antennas in the array antenna. Assuming that the vehicle is moving on a horizontal flat surface so that the roll and pitch of the user frame remain unchanged in terms of the reference frame, if the azimuth is equal to $\alpha_{U}$, the relationship between the user frame and the reference frame is given by [12]

$$
\left[\begin{array}{l}
x_{U} \\
y_{U}
\end{array}\right]=\left[\begin{array}{cc}
\cos \left(\alpha_{U}\right) & \sin \left(\alpha_{U}\right) \\
-\sin \left(\alpha_{U}\right) & \cos \left(\alpha_{U}\right)
\end{array}\right]\left[\begin{array}{l}
x_{R} \\
y_{R}
\end{array}\right] .
$$

Hence, the FLAOA measurement is represented by

$$
\begin{aligned}
\tan \left(\varphi_{i}\right) & =\frac{y_{U}-y_{U i}}{x_{U}-x_{U i}} \\
& =\frac{-\left(x-x_{i}\right) \sin \left(\alpha_{U}\right)+\left(y-y_{i}\right) \cos \left(\alpha_{U}\right)}{\left(x-x_{i}\right) \cos \left(\alpha_{U}\right)+\left(y-y_{i}\right) \sin \left(\alpha_{U}\right)} \\
& =\frac{-\left(x-x_{i}\right) \tan \left(\alpha_{U}\right)+\left(y-y_{i}\right)}{\left(x-x_{i}\right)+\left(y-y_{i}\right) \tan \left(\alpha_{U}\right)}
\end{aligned}
$$

where $\left(x_{U}, y_{U}\right)$ and $\left(x_{U i}, y_{U i}\right)$ denote the user and the $i$ th sensor position with respect to the user frame, respectively. 


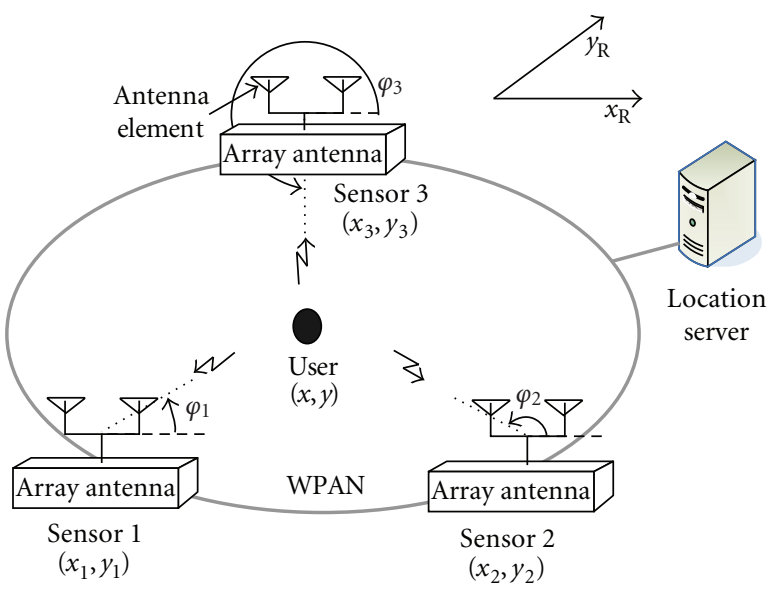

(a) RLAOA positioning

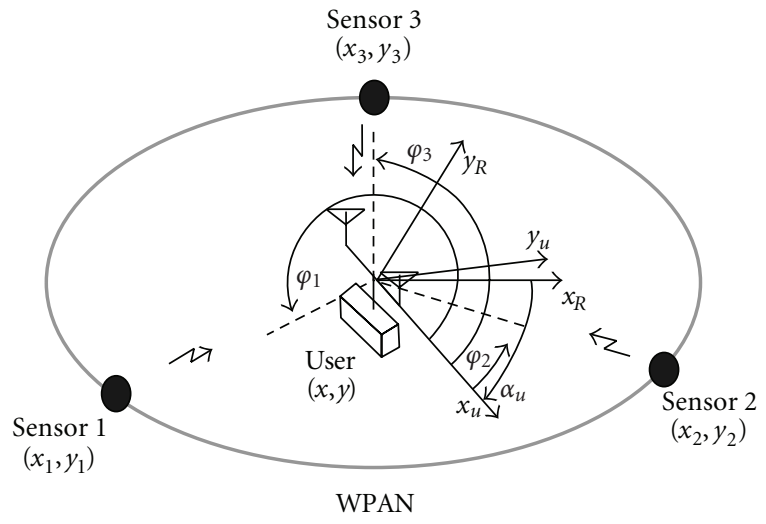

(b) FLAOA positioning

Figure 2: Basic principles of RLAOA and FLAOA.

Assuming that the angle measurement noise $n_{\varphi i}$ is IID (independent and identically distributed) white Gaussian noise with a variance of $\sigma_{\varphi}^{2}$, the FLAOA measurement equation considering the noise is written as

$$
\begin{array}{r}
z_{\varphi i}=\varphi_{i}+n_{\varphi i} \\
=\tan ^{-1}\left(\frac{-\left(x-x_{i}\right) \tan \left(\alpha_{U}\right)+\left(y-y_{i}\right)}{\left(x-x_{i}\right)+\left(y-y_{i}\right) \tan \left(\alpha_{U}\right)}\right)+n_{\varphi i}, \\
i=1, \ldots, m,
\end{array}
$$

where $m$ is the number of sensors. In (5), the FLAOA measurement is influenced by the azimuth $\alpha_{U}$ as well as by the user position. Therefore, at least three measurements are theoretically required to estimate the azimuth and the position in two-dimensional FLAOA positioning.

2.2. Gauss-Newton Method for FLAOA Measurement. The GN method has been widely used in various radionavigation systems using TOA, TDOA, or RLAOA measurements $[3,5,6]$. In the GN method, linearization is done via Taylor series expansion, and position estimates are obtained using ILS (iterated least square) or IWLS (iterated weighted least square) [13]. In this section, the GN method using FLAOA measurements is derived.

Define $r=\tan \alpha_{U}$ and $\underline{x}=\left[\begin{array}{lll}x & y & r\end{array}\right]^{T}$. Using a Taylor series, $\varphi_{i}$ in (5) is linearized as

$$
\begin{aligned}
\varphi_{i}(\underline{x}) \cong & \varphi_{i}\left(\underline{x}_{0}\right)+\left.\frac{\partial \varphi_{i}}{\partial x}\right|_{\underline{\mathbf{x}}_{=}=\underline{\mathbf{x}}_{0}}\left(x-x_{0}\right) \\
& +\left.\frac{\partial \varphi_{i}}{\partial y}\right|_{\underline{\underline{\mathbf{x}}=\underline{\mathbf{x}}_{0}}}\left(y-y_{0}\right)+\left.\frac{\partial \varphi_{i}}{\partial r}\right|_{\underline{\mathbf{x}}_{=}=\underline{\mathbf{x}}_{0}}\left(r-r_{0}\right),
\end{aligned}
$$

where $\underline{x}_{0}=\left[\begin{array}{lll}x_{0} & y_{0} & r_{0}\end{array}\right]^{T}$ is a nominal vector. From (5) and (6), the linearized measurement equation is obtained by

$$
\begin{aligned}
& \delta \underline{\mathrm{z}}_{\varphi} \equiv\left[\begin{array}{c}
\delta z_{\varphi 1}(\underline{x}) \\
\vdots \\
\delta z_{\varphi m}(\underline{x})
\end{array}\right]=\left[\begin{array}{c}
z_{\varphi 1}(\underline{x})-\varphi_{1}\left(\underline{x}_{0}\right) \\
\vdots \\
z_{\varphi m}(\underline{x})-\varphi_{m}\left(\underline{x}_{0}\right)
\end{array}\right]
\end{aligned}
$$

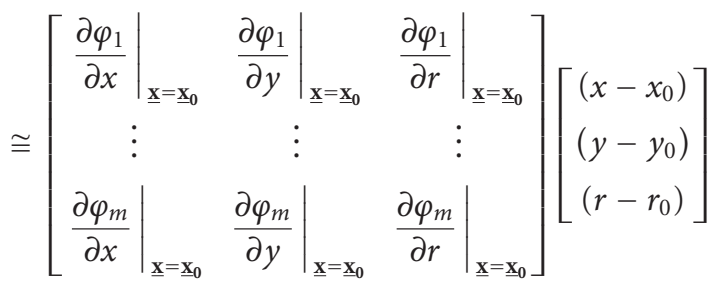

$$
\begin{aligned}
& +\left[\begin{array}{c}
n_{\varphi 1} \\
\vdots \\
n_{\varphi m}
\end{array}\right] \equiv G\left(\underline{x}-\underline{x}_{0}\right)+\underline{n} .
\end{aligned}
$$

With the linearized measurement of (7), in the GN method, position estimates are iteratively obtained by applying ILS. Here, the prior estimate is used as a nominal point in next iteration. In most cases, the estimate obtained by the GN method may converge to a true value within 3-5 iterations. When the initial point is not properly chosen or when the sensor geometry is poor, however, the estimate can converge to an erroneous value or even diverge.

\subsection{A Closed-Form Solution for FLAOA Measurement.} RLAOA measurement is nonlinear to user position as in (2). By measurement transformation, a closed-form solution for RLAOA measurement was derived in [7]. As FLAOA measurement is influenced by the azimuth of vehicle as well as by its position, a closed-form solution for FLAOA measurement should be newly proposed. In this section, FLAOA positioning using the closed-form solution is derived by employing parameter transformation in addition to measurement transformation.

Equation (4) can be rewritten as

$$
\begin{gathered}
\left(y-y_{i}\right) \tan \left(\varphi_{i}\right) \tan \left(\alpha_{U}\right)+\left(x-x_{i}\right) \tan \left(\varphi_{i}\right) \\
=\left(y-y_{i}\right)-\left(x-x_{i}\right) \tan \left(\alpha_{U}\right)
\end{gathered}
$$


Defining $r=\tan \left(\alpha_{U}\right), p=x+r y$, and $q=r x-y$ and using (5), (8) is rewritten as

$$
\begin{gathered}
p \tan \left(z_{\varphi i}-n_{\varphi i}\right)+q-r\left\{y_{i} \tan \left(z_{\varphi i}-n_{\varphi i}\right)+x_{i}\right\} \\
=x_{i} \tan \left(z_{\varphi i}-n_{\varphi i}\right)-y_{i} .
\end{gathered}
$$

If $n_{\varphi i}$ is sufficiently small, using the fact that $\tan \left(z_{\varphi i}-\right.$ $\left.n_{\varphi i}\right) \cong\left\{\sin \left(z_{\varphi i}\right)-\cos \left(z_{\varphi i}\right) n_{\varphi i}\right\} /\left\{\cos \left(z_{\varphi i}\right)+\sin \left(z_{\varphi i}\right) n_{\varphi i}\right\},(9)$ is approximated as

$$
\begin{gathered}
\left(p-r y_{i}\right)\left\{\tan \left(z_{\varphi i}\right)-n_{\varphi i}\right\}+\left(q-r x_{i}\right)\left\{1+n_{\varphi i} \tan \left(z_{\varphi i}\right)\right\} \\
\cong x_{i}\left\{\tan \left(z_{\varphi i}\right)-n_{\varphi i}\right\}-y_{i}\left\{1+n_{\varphi i} \tan \left(z_{\varphi i}\right)\right\} .
\end{gathered}
$$

For $i=1, \ldots, m,(10)$ is represented by a matrix-vector form that is given by

$$
\begin{aligned}
\underline{z}_{\varphi}{ }^{\prime} \equiv & {\left[\begin{array}{ccc}
x_{1} \tan \left(z_{\varphi 1}\right)-y_{1} \\
\vdots & \\
x_{m} \tan \left(z_{\varphi m}\right)-y_{m}
\end{array}\right] } \\
= & {\left[\begin{array}{ccc}
\tan \left(z_{\varphi 1}\right) & 1 & -\left\{y_{1} \tan \left(z_{\varphi 1}\right)+x_{1}\right\} \\
\vdots & \vdots & \\
\tan \left(z_{\varphi m}\right) & 1 & -\left\{y_{m} \tan \left(z_{\varphi m}\right)+x_{m}\right\}
\end{array}\right]\left[\begin{array}{c}
p \\
q \\
r
\end{array}\right] } \\
& +\left[\begin{array}{cccc}
k_{1} & 0 & \ldots & 0 \\
0 & k_{2} & & \vdots \\
\vdots & & \ddots & 0 \\
0 & \ldots & 0 & k_{m}
\end{array}\right]\left[\begin{array}{c}
n_{\varphi 1} \\
\vdots \\
n_{\varphi m}
\end{array}\right] \\
\equiv & H \underline{x^{\prime}}+J \underline{n}=H \underline{x}^{\prime}+\underline{v},
\end{aligned}
$$

where $k_{i}=r y_{i}-p+x_{i}+\left(q-r x_{i}+y_{i}\right) \tan \left(z_{\varphi i}\right)$.

As $n_{\varphi i}$ in (5) is IID white Gaussian noise with a variance of $\sigma_{\varphi}^{2}$, the covariance matrix of the pseudomeasurement noise is equal to $Q=\operatorname{cov}(\underline{v})=\sigma_{\varphi}^{2}\left(J \cdot J^{T}\right)$. When $\underline{x}^{\prime}$ is estimated using WLS (weighted least square), weighting matrix is typically given by $Q^{-1}$. As $p, q$, and $r$ in $Q$ are unknown, a two-step estimation technique is used, as in [10]. In the first step, using the LS (least square), a primary estimate of $\underline{x}^{\prime}$ is obtained to estimate $\operatorname{cov}(\underline{v})$ :

$$
\underline{\hat{x}}_{\mathrm{LS}}^{\prime}=\left(H^{T} H\right)^{-1} H^{T} \underline{z}_{\varphi}{ }^{\prime} .
$$

In the second step, with the estimated weighting matrix $\hat{Q}$, the final estimate of $\underline{x}^{\prime}$ is obtained using the WLS as given by

$$
\underline{\hat{x}}^{\prime}=\left[H^{T} \hat{Q}^{-1} H\right]^{-1} H^{T} \hat{Q}^{-1} \underline{z}_{\varphi}{ }^{\prime} .
$$

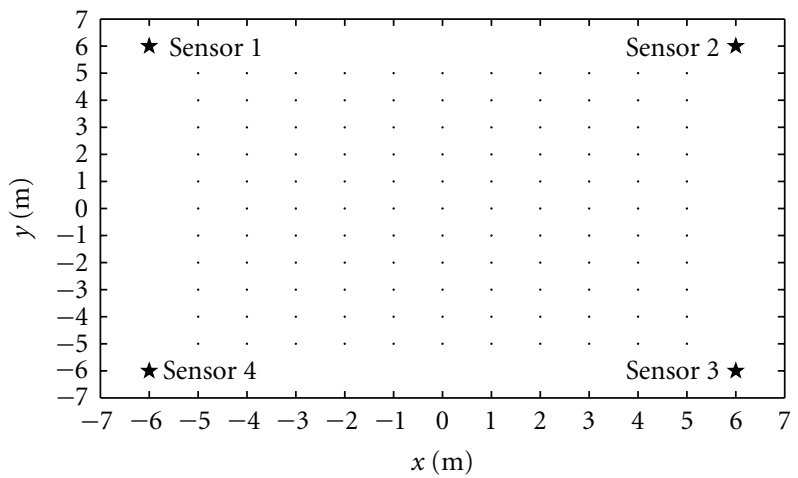

FIGURE 3: Sensor allocation and reference points.

From $\underline{\hat{x}}^{\prime}=[\hat{p} \hat{q} \hat{r}]^{T}$ in (13), estimates of the user position and the azimuth are represented as

$$
\underline{\underline{x}}=\left[\begin{array}{c}
\hat{x} \\
\hat{y} \\
\hat{\alpha}_{U}
\end{array}\right]=\left[\begin{array}{c}
\frac{\hat{p}+\hat{q} \hat{r}}{1+\hat{r}^{2}} \\
\hat{p} \hat{r}-\hat{q} \\
\frac{1+\hat{r}^{2}}{\tan ^{-1}(\hat{r})}
\end{array}\right] .
$$

As the closed-form solution for FLAOA measurements has a noniterative structure, it is not associated with a divergence problem, and computation is simple compared to the GN method.

\section{Simulation Results}

The performance of the FLAOA positioning scheme is compared to that of RLAOA positioning in a computer simulation. It is assumed that a mobile vehicle is working indoors and that it moves on a horizontal flat workspace whose size is $12 \mathrm{~m} \times 12 \mathrm{~m}$. The work space was determined based on the Institute of Electrical and Electronics Engineering (IEEE) 802.15.4a standard for low rate WPAN [14]. It is also assumed that angle measurements are obtained using IR-UWB system with linear array antennas for RLAOA. For FLAOA, an "L" shape planar array antenna is used to avoid angle ambiguity. Considering the typical accuracy of IRUWB location, the variance of the angle measurement noise $n_{\varphi i}$ in (5) is given as $\sigma_{\varphi}^{2}=10^{-3} \mathrm{rad}^{2}$ [15]. As the IR-UWB system is resistant to severe multipath and NLOS (nonlineof-sight) problem is not a topic of this paper, NLOS or multipath is not considered in the simulation. Four sensors are symmetrically allocated, as shown in Figure 3. In the figure, the sensors are denoted by " $\star$ ". It is known that the positioning accuracy of radio-navigation varies depending on the sensor geometry with respect to the user position [16]. To analyze the impact of the sensor geometry on the accuracy of the positioning, 121 reference points were selected. They are denoted as "•" in Figure 3. 100 trials were executed at each reference point to obtain the statistical data.

The performance of FLAOA positioning using the GN method was analyzed first. In the simulation, an initial position estimate is given by $(0,0)$, and iteration is set to 


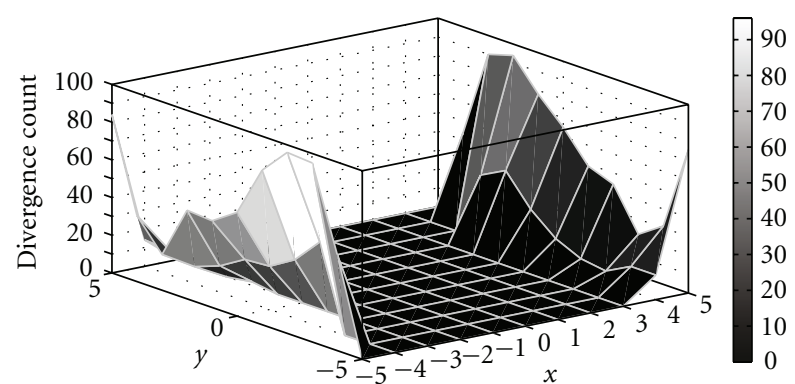

(a) FLAOA

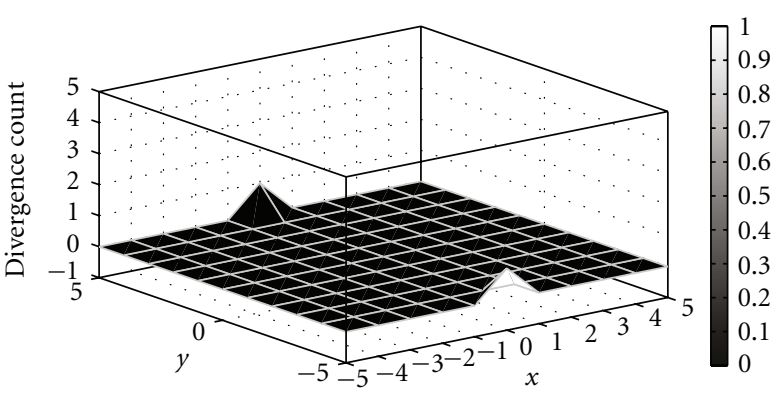

(b) RLAOA

FIgURE 4: Number of divergence in the GN method.

be terminated when the estimate converges to within $1 \mathrm{~cm}$. Figure 4 shows the number of divergence trials in the GN method for each reference point. In FLAOA positioning, the azimuth of vehicle is given as $\alpha_{U}=-10^{\circ}$. At 37 reference points near the border of the workspace, divergence takes place in FLAOA positioning using the GN method. On the other hand, in RLAOA using the GN method, divergence occurs only at two reference points. At the reference points where divergence occurs, the relative frequency is .09-0.96 in FLAOA positioning, whereas it is 0.01 in RLAOA. The relative frequency is defined as the number of divergence out of total trials. In addition, the performance of FLAOA positioning using the GN method is greatly influenced by the azimuth $\alpha_{U}$, in other words, the geometry seen from the vehicle's array antenna. As divergence occurs in a large part of the workspace and because the relative frequency is excessively high, FLAOA positioning using the GN method is inadequate for application to localization issues.

Next, the performance of FLAOA positioning using the closed-form solution is analyzed. Three location estimation methods are compared: the proposed FLAOA positioning method, RLAOA using the closed-form solution as proposed in [10], and RLAOA using the GN method. Figure 5 shows the range estimation error of the three methods at each reference point. In Figure 5(a), the azimuth of vehicle is given as $\alpha_{U}=-10^{\circ}$. In the GN method, the trials whose solutions diverge are excluded from the plot. The range estimation error is obtained from

$$
e_{\hat{r}}=E\left\{\sqrt{(x-\hat{x})^{2}+(y-\hat{y})^{2}}\right\}
$$

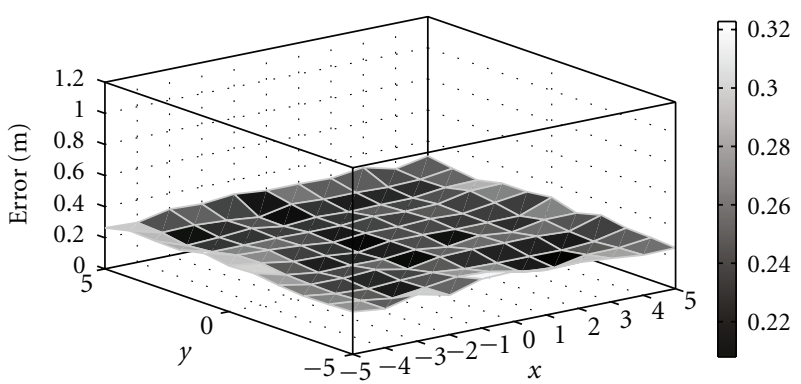

(a) FLAOA using the closed-form solution

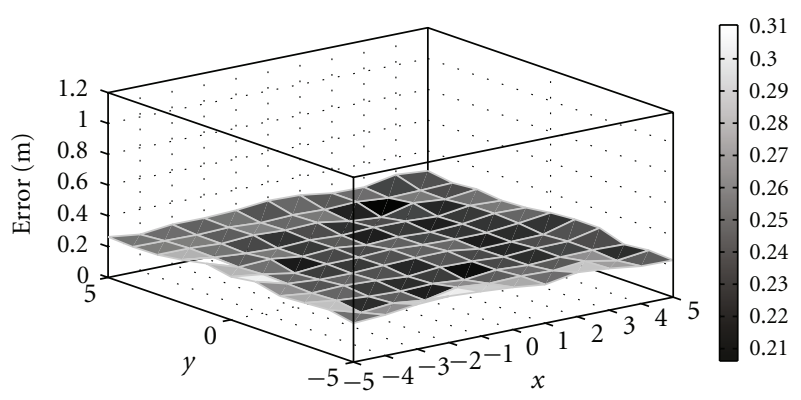

(b) RLAOA using the closed-form solution

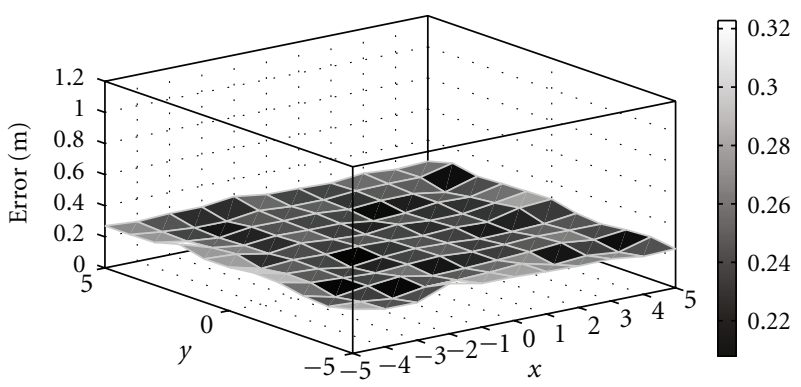

(c) RLAOA using the GN method

FIgURE 5: Range estimation error.

In the two RLAOA positioning methods, the range estimation error is $0.2 \sim 0.31 \mathrm{~m}$ over the entire workspace. In the proposed FLAOA method, the range estimation error is $0.21-0.32 \mathrm{~m}$, which is nearly identical to that with RLAOA positioning.

As mentioned earlier, azimuth estimate is also obtained in FLAOA positioning. Figure 6 shows the RMS (root mean square) azimuth estimation error at each reference point in the proposed FLAOA positioning with different azimuth angles. For the entire workspace, the angle estimation error is only $0.75-1.16^{\circ}$ in all three cases. Considering $\sigma_{\varphi} \cong 1.81^{\circ}$, this is sufficiently accurate for use in guidance control of indoor mobile vehicles. Except when the azimuth approaches $\alpha_{U}= \pm 90^{\circ}$, the magnitude of the estimation error is consistent regardless of the azimuth angle. At $\alpha_{U}= \pm 90^{\circ}$, $r=\tan \left(\alpha_{U}\right)$ in $\underline{x}^{\prime}$ of (11) goes to infinity. Since "L" shape planar antenna is used in FLAOA, this singularity problem can be avoided. As with the azimuth estimation error, the range estimation error is consistent regardless of the azimuth angle, except when the azimuth approaches $\alpha_{U}= \pm 90^{\circ}$. 


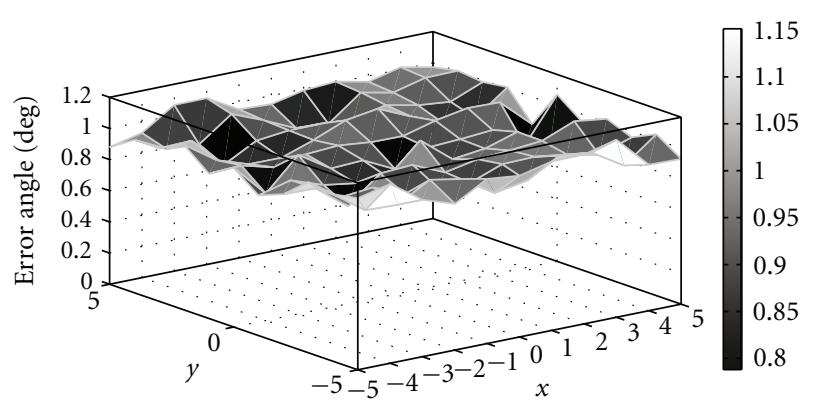

(a) $\alpha_{U}=-10^{\circ}$

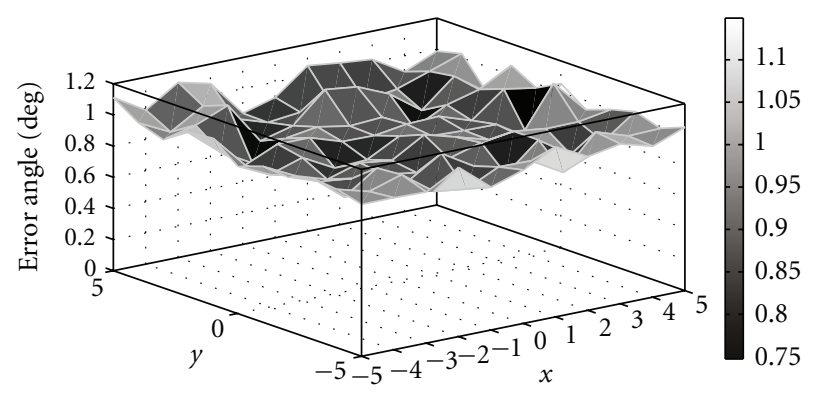

(b) $\alpha_{U}=-45^{\circ}$

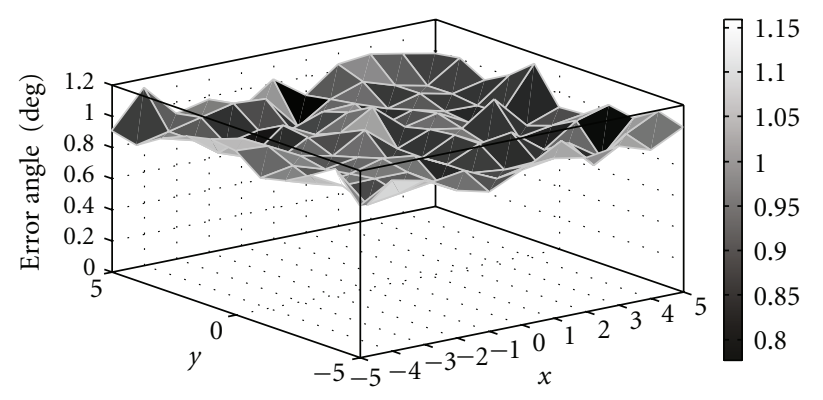

(c) $\alpha_{U}=-80^{\circ}$

FIGURe 6: RMS azimuth estimation error in FLAOA positioning using the closed-form solution.

\section{Conclusions}

This paper presents a new angle-based location method using FLAOA measurements. Two types of position estimation methods using FLAOA measurements are examined: the GN method and the closed-form solution. Simulation results showed that FLAOA positioning using the GN method diverges by more than $30 \%$ of the workspace and that it is inadequate for localization of mobile vehicles. On the other hand, the positioning performance of FLAOA using the closed-form solution is nearly identical to that of conventional RLAOA. Moreover, the proposed method provides an azimuth estimate that is sufficiently accurate for use in guidance control.

Though FLAOA positioning requires one additional sensor compared to RLAOA, it can provide an azimuth that is valuable when used with guidance control of mobile vehicles. In FLAOA positioning, it is also possible to make sensors small and light. Therefore, the proposed FLAOA positioning method is a potential solution for applications related to the localization and guidance of mobile vehicles.

\section{References}

[1] K. D. McDonald, "The modernization of GPS: plans, new capabilities and the future relationship to Galileo," Journal of Global Positioning Systems, vol. 1, no. 1, pp. 1-17, 2002.

[2] Y. Zhao, "Mobile phone location determination and its impact on intelligent transportation systems," IEEE Transactions on Intelligent Transportation Systems, vol. 1, no. 1, pp. 55-64, 2000.

[3] I. Oppermann, M. Hämäläinen, and J. Iinatti, UWB Theory and Applications, John Willy \& Sons, Chichester, UK, 2004.

[4] T. S. Rappaport, J. H. Reed, and B. D. Woerner, "Position location using wireless communications on highways of the future," IEEE Communications Magazine, vol. 34, no. 10, pp. 33-41, 1996.

[5] W. H. Foy, "Position-location solutions by Taylor-series estimation," IEEE Transactions on Aerospace and Electronic Systems, vol. 12, no. 2, pp. 187-194, 1976.

[6] D. J. Torrieri, "Statistical theory of passive location systems," IEEE Transactions on Aerospace and Electronic Systems, vol. 20, no. 2, pp. 183-198, 1984.

[7] K. W. Cheung, H. C. So, W.-K. Ma, and Y. T. Chan, "A constrained least squares approach to mobile positioning: algorithms and optimality," EURASIP Journal on Applied Signal Processing, vol. 2006, Article ID 20858, 23 pages, 2006.

[8] S. M. Kay, Fundamentals of Statistical Signal Processing: Estimation Theory, Prentice-Hall, Englewood Cliffs, NJ, USA, 1993.

[9] A. Pagès-Zamora, J. Vidal, and D. H. Brooks, "Closed-form solution for positioning based on angle of arrival measurements," in Proceedings of 13th IEEE International Symposium on Personal, Indoor and Mobile Radio Communication (PIMRC '02), vol. 4, pp. 1522-1526, Lisboa, Portugal, September 2002.

[10] D.-H. Kim, S.-H. Lee, K.-S. Park, and T.-K. Sung, "Development of an AOA location method using covariance estimation," in Proceedings of the 4th IASTED Asian Conference on Communication Systems and Networks (AsiaCSN '07), pp. 1418, Phuket, Thailand, April 2007.

[11] B. Forssell, Radionavigation Systems, Artech House, Norwood, Mass, USA, 2008.

[12] R. M. Rogers, Applied Mathematics in Integrated Navigation Systems, AIAA, Reston, Va, USA, 2000.

[13] J. M. Mendel, Lessons in Estimation Theory for Signal Processing, Communications, and Control, Prentice-Hall, Upper Saddle River, NJ, USA, 1995.

[14] IEEE Standard for Information technology, Telecommunications and information exchange between systems, Local and metropolitan area networks, Specific requirements; Part 15.4: Wireless Medium Access Control (MAC) and Physical Layer (PHY) Specifications for Low-Rate Wireless Personal Area Networks (WPANs), IEEE Standard, August 2007.

[15] Ubisense System overview, June 2009, http://www.ubisense .net/.

[16] E. D. Kaplan, Understanding GPS Principles and Applications, Artech House, Norwood, Mass, USA, 1996. 